\title{
Nonnegative Matrix Factor 2-D Deconvolution for Blind Single Channel Source Separation
}

\author{
Mikkel N. Schmidt and Morten Mørup \\ Technical University of Denmark \\ Informatics and Mathematical Modelling \\ Richard Petersens Plads, Building 321 \\ DK-2800 Kgs. Lyngby, Denmark \\ \{mns, mm\}@imm.dtu.dk
}

\begin{abstract}
We present a novel method for blind separation of instruments in polyphonic music based on a non-negative matrix factor 2-D deconvolution algorithm. Using a model which is convolutive in both time and frequency we factorize a spectrogram representation of music into components corresponding to individual instruments. Based on this factorization we separate the instruments using spectrogram masking. The proposed algorithm has applications in computational auditory scene analysis, music information retrieval, and automatic music transcription.
\end{abstract}

\section{Introduction}

The separation of multiple sound sources from a single channel recording is a difficult problem which has been extensively addressed in the literature. Many of the proposed methods are based on matrix decompositions of a spectrogram representation of the sound. The basic idea is to represent the sources by different frequency signatures which vary in intensity over time.

Non-negative matrix factorization (NMF) $[1,2]$ has been proven a very useful tool in a variety of signal processing fields. NMF gives a sparse (or parts-based) decomposition [2] and under certain conditions the decomposition is unique [3] making it unnecessary to impose constraints in the form of orthogonality or independence. Efficient algorithms for computing the NMF have been introduced by Lee and Seung [4]. NMF has a variety of applications in music signal processing; recently, Helén and Virtanen [5] described a method for separating drums from polyphonic music using NMF and Smaragdis and Brown [6] used NMF for automatic transcription of polyphonic music.

When polyphonic music is modelled by factorizing the magnitude spectrogram with NMF, each instrument is modelled by an instantaneous frequency signature which can vary over time. Smaragdis [7] introduced an extension to NMF, namely the non-negative matrix factor deconvolution (NMFD) algorithm in which each instrument is modelled by a time-frequency signature which varies in intensity over time. Thus, the model can represent components with temporal structure. Smaragdis showed how this can be used to separate individual drums 
from a real recording of drum sounds. This approach was further pursued by Wang and Plumley [8] who separated mixtures of different musical instruments. Virtanen [9] presented an algorithm based on similar ideas and evaluated its performance by separating mixtures of harmonic sounds.

In this paper, we propose a new method to factorize a log-frequency spectrogram using a model which can represent both temporal structure and the pitch change which occurs when an instrument plays different notes. We use a log-frequency spectrogram such that a pitch change corresponds to a displacement on the frequency axis. We denote this the non-negative matrix factor 2-D deconvolution (NMF2D). Where previous methods needed one component to model each note for each instrument, the proposed model represents each instrument compactly by a single time-frequency profile convolved in both time and frequency by a time-pitch weight matrix. This model dramatically decreases the number of components needed to model various instruments and effectively solves the blind single channel source separation problem for certain classes of musical signals. In section 2 we introduce the NMF2D model and derive the update equations for recursively computing the factorization based on two different cost functions. In section 3 we show how the algorithm can be used to analyze and separate polyphonic music and we compare the algorithm to the NMFD method of Smaragdis [7]. This is followed by a discussion of the results.

\section{Method}

Consider the non-negative matrix factorization problem:

$$
\mathbf{V} \approx \mathbf{W H},
$$

where $\mathbf{V}, \mathbf{W}$, and $\mathbf{H}$ are non-negative matrices. Lee and Seung [4] devise two algorithms to find $\mathbf{W}$ and $\mathbf{H}$ : For the least square error and the KL divergence they show that the following recursive updates converge to a local minimum:

$$
\begin{array}{rll}
\text { Least square error : } & \mathbf{W} \leftarrow \mathbf{W} \bullet \frac{\mathbf{V H}^{T}}{\mathbf{W H H}^{T}}, & \mathbf{H} \leftarrow \mathbf{H} \bullet \frac{\mathbf{W}^{T} \mathbf{V}}{\mathbf{W}^{T} \mathbf{W H}}, \\
\text { KL divergence : } & \mathbf{W} \leftarrow \mathbf{W} \bullet \frac{\frac{\mathbf{V}}{\mathbf{W H}} \mathbf{H}^{T}}{\mathbf{1} \cdot \mathbf{H}}, & \mathbf{H} \leftarrow \mathbf{H} \bullet \frac{\mathbf{W}^{T} \mathbf{v}}{\mathbf{W} \cdot \mathbf{H}},
\end{array}
$$

where $A \bullet B$ denotes element-wise multiplication and $\frac{A}{B}$ denotes element-wise division. These algorithms can be derived by minimizing the cost function using gradient descent and choosing the stepsize appropriately to yield simple multiplicative updates.

We now extend the NMF model to be a 2-dimensional convolution of $\mathbf{W}^{\tau}$ which depends on time, $\tau$, and $\mathbf{H}^{\phi}$ which depends on pitch, $\phi$. This forms the non-negative factor 2-D deconvolution (NMF2D) model:

$$
\mathbf{V} \approx \boldsymbol{\Lambda}=\sum_{\tau} \sum_{\phi} \underset{\mathbf{W}^{\tau}}{\stackrel{\downarrow \phi}{\mathbf{H}^{\phi}}}
$$


where $\downarrow \phi$ denotes the downward shift operator which moves each element in the matrix $\phi$ rows down, and $\rightarrow \tau$ denotes the right shift operator which moves each element in the matrix $\tau$ columns to the right, i.e.:

$$
\mathbf{A}=\left(\begin{array}{lll}
1 & 2 & 3 \\
4 & 5 & 6 \\
7 & 8 & 9
\end{array}\right), \quad \stackrel{\downarrow}{\mathbf{A}}=\left(\begin{array}{lll}
0 & 0 & 0 \\
0 & 0 & 0 \\
1 & 2 & 3
\end{array}\right), \quad \overrightarrow{\mathbf{A}}=\left(\begin{array}{lll}
0 & 1 & 2 \\
0 & 4 & 5 \\
0 & 7 & 8
\end{array}\right)
$$

We note that the NMFD model introduced by Smaragdis [7] is a special case of the NMF2D model where $\phi=\{0\}$.

Each element in $\boldsymbol{\Lambda}$ is given by

$$
\boldsymbol{\Lambda}_{i, j}=\sum_{\tau} \sum_{\phi} \sum_{d} \mathbf{W}_{i-\phi, d}^{\tau} \mathbf{H}_{d, j-\tau}^{\phi}
$$

In the following derivation of the update steps required to compute $\mathbf{W}^{\tau}$ and $\mathbf{H}^{\phi}$ we will need the derivative of a given element $\boldsymbol{\Lambda}_{i, j}$ with respect to a given element $\mathbf{W}_{k, d}^{\tau}$ :

$$
\begin{aligned}
\frac{\partial \boldsymbol{\Lambda}_{i, j}}{\partial \mathbf{W}_{k, d}^{\tau}} & =\frac{\partial}{\partial \mathbf{W}_{k, d}^{\tau}}\left(\sum_{\tau} \sum_{\phi} \sum_{d} \mathbf{W}_{i-\phi, d}^{\tau} \mathbf{H}_{d, j-\tau}^{\phi}\right) \\
& =\frac{\partial}{\partial \mathbf{W}_{k, d}^{\tau}}\left(\sum_{\phi} \mathbf{W}_{i-\phi, d}^{\tau} \mathbf{H}_{d, j-\tau}^{\phi}\right) \\
& =\left\{\begin{array}{cc}
\mathbf{H}_{d, j-\tau}^{\phi} & \phi=i-k \\
0 & \text { otherwise. }
\end{array}\right.
\end{aligned}
$$

\subsection{NMF2D Least Squares}

Now, we consider the least squares cost function which corresponds to maximizing the likelihood of a gaussian noise model:

$$
C_{L S}=\|\mathbf{V}-\boldsymbol{\Lambda}\|_{f}^{2}=\sum_{i} \sum_{j}\left(\mathbf{V}_{i, j}-\boldsymbol{\Lambda}_{i, j}\right)^{2} .
$$

Differentiating $C_{L S}$ with respect to a given element in $\mathbf{W}^{\tau}$ gives:

$$
\begin{aligned}
\frac{\partial C_{L S}}{\partial \mathbf{W}_{k, d}^{\tau}} & =\frac{\partial}{\partial \mathbf{W}_{k, d}^{\tau}} \sum_{i} \sum_{j}\left(\mathbf{V}_{i, j}-\boldsymbol{\Lambda}_{i, j}\right)^{2} \\
& =-2 \sum_{i} \sum_{j}\left(\mathbf{V}_{i, j}-\boldsymbol{\Lambda}_{i, j}\right) \frac{\partial \boldsymbol{\Lambda}_{i, j}}{\partial \mathbf{W}_{k, d}^{\tau}} \\
& =-2 \sum_{\phi} \sum_{j}\left(\mathbf{V}_{\phi+k, j}-\boldsymbol{\Lambda}_{\phi+k, j}\right) \mathbf{H}_{d, j-\tau}^{\phi} .
\end{aligned}
$$


The recursive update steps for the gradient descent are given by:

$$
\mathbf{W}_{k, d}^{\tau} \leftarrow \mathbf{W}_{k, d}^{\tau}-\eta \frac{\partial C_{L S}}{\partial \mathbf{W}_{k, d}^{\tau}} .
$$

Similar to the approach of Lee and Seung [4], we choose the step size $\eta$ so that the first term in (12) is canceled:

$$
\eta=\frac{\mathbf{W}_{k, d}^{\tau}}{-2 \sum_{\phi} \sum_{j} \boldsymbol{\Lambda}_{\phi+k, j} \mathbf{H}_{d, j-\tau}^{\phi}},
$$

which gives us the following simple multiplicative updates:

$$
\mathbf{W}_{k, d}^{\tau} \leftarrow \mathbf{W}_{k, d}^{\tau} \frac{\sum_{\phi} \sum_{j} \mathbf{V}_{\phi+k, j} \mathbf{H}_{d, j-\tau}^{\phi}}{\sum_{\phi} \sum_{j} \boldsymbol{\Lambda}_{\phi+k, j} \mathbf{H}_{d, j-\tau}^{\phi}} .
$$

By noticing that transposing equation (3) interchanges the order of $\mathbf{W}^{\tau}$ and $\mathbf{H}^{\phi}$ in the model, the updates of $\mathbf{H}^{\phi}$ can easily be found. In matrix notation the updates can be written as:

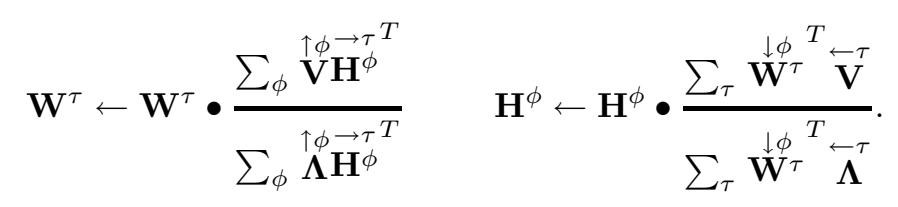

\subsection{NMF2D by KL Divergence}

Consider the Kullbach-Leibler (KL) divergence given by:

$$
C_{K L}=\sum_{i} \sum_{j} \mathbf{V}_{i, j} \log \frac{\mathbf{V}_{i, j}}{\boldsymbol{\Lambda}_{i, j}}-\mathbf{V}_{i, j}+\boldsymbol{\Lambda}_{i, j}
$$

Minimizing the KL divergence corresponds to assuming multinomial noise model. Differentiating this cost function with respect to a given element in $\mathbf{W}^{\tau}$ gives:

$$
\begin{aligned}
\frac{\partial C_{K L}}{\partial \mathbf{W}_{k, d}^{\tau}} & =\frac{\partial}{\partial \mathbf{W}_{k, d}^{\tau}} \sum_{i} \sum_{j} \mathbf{V}_{i, j} \log \frac{\mathbf{V}_{i, j}}{\boldsymbol{\Lambda}_{i, j}}-\mathbf{V}_{i, j}+\boldsymbol{\Lambda}_{i, j} \\
& =\sum_{i} \sum_{j}\left(1-\frac{\mathbf{V}_{i, j}}{\boldsymbol{\Lambda}_{i, j}}\right) \frac{\partial \boldsymbol{\Lambda}_{i, j}}{\partial \mathbf{W}_{k, d}^{\tau}} \\
& =\sum_{\phi} \sum_{j}\left(1-\frac{\mathbf{V}_{k+\phi, j}}{\boldsymbol{\Lambda}_{k+\phi, j}}\right) \mathbf{H}_{d, j-\tau}^{\phi} .
\end{aligned}
$$

Again, the recursive gradient descent update steps are given by:

$$
\mathbf{W}_{k, d}^{\tau} \leftarrow \mathbf{W}_{k, d}^{\tau}-\eta \frac{\partial C_{K L}}{\partial \mathbf{W}_{k, d}^{\tau}},
$$


and the step size $\eta$ is chosen so that the first term in equation (20) is canceled:

$$
\eta=\frac{\mathbf{W}_{k, d}^{\tau}}{\sum_{\phi} \sum_{j} \mathbf{H}_{d, j-\tau}^{\phi}},
$$

which gives the following simple multiplicative updates:

$$
\mathbf{W}_{k, d}^{\tau} \leftarrow \mathbf{W}_{k, d}^{\tau} \frac{\sum_{\phi} \sum_{j} \frac{\mathbf{V}_{\phi+k, j}}{\boldsymbol{\Lambda}_{\phi+k, j}} \mathbf{H}_{d, j-\tau}^{\phi}}{\sum_{\phi} \sum_{j} \mathbf{H}_{d, j-\tau}^{\phi}} .
$$

Again, the updates for $\mathbf{H}^{\phi}$ can easily be found by symmetry, and the updates can be written in matrix notation as:

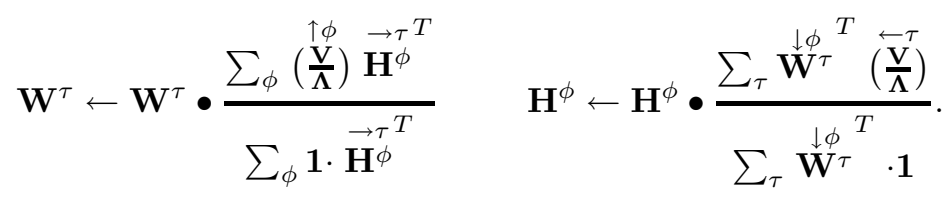

\section{Experimental Results}

In order to demonstrate our NMF2D algorithm, we have analyzed a 4 second piece of computer generated polyphonic music containing a trumpet and a piano. For comparison we have also analyzed the same piece of music by the NMFD algorithm [7]. For both algorithms we used the least squares cost function. The score of the piece of music is shown in Fig. 1. The trumpet and the piano play a different short melodic passage each consisting of three distinct notes. We generated the music at a sample rate of $16 \mathrm{kHz}$ and analyzed it by the short time Fourier transform with a 2048 point Hanning windowed FFT and 50\% overlap. This gave us 63 FFT slices. We grouped the spectrogram bins into 175 logarithmically spaced frequency bins in the range of $50 \mathrm{~Hz}$ to $8 \mathrm{kHz}$ with 24 bins per octave, which correponds to twice the resolution of the equal tempered musical scale. Then, we performed the NMF2D and NMFD factorization of the log-frequency magnitude spectrogram.

For the NMF2D we used two factors, $d=2$, since we seek to separate two instruments. We empirically chose to use seven convolutive components in time, $\tau=\{0, \ldots, 6\}$, corresponding to approximatly $45 \mathrm{~ms}$. The pitch of the notes played in the music span three whole notes. Consequently, we chose to use 12 convolutive components in pitch, i.e. $\phi=\{0, \ldots, 11\}$.

For the NMFD we used six factors, $d=6$, corresponding to the total number of different tones played by the two instruments. Similar to the experiment with NMF2D we used seven convolutive components in time. For the experiment with NMFD we used our formulation of the NMF2D algorithm with $\phi=\{0\}$, since the NMFD is a special case of the NMF2D algorithm. 
The results of the experiments with NMFD and NMF2D are shown in Fig. 2 and Fig. 3 respectively. The NMFD algorithm factorized each individual note from each instrument into a separate component, whereas the NMF2D algorithm factorized each instrument into a separate component.

We used the NMF2D factorization of the music to reconstruct the individual instruments separately by spectrogram masking. First, we reconstructed the spectrum of each individual instrument by computing equation (4) for each specific value of $d$. Based on these reconstructed individual instrument spectra we constructed a spectrogram mask for each instrument, so that each spectrogram bin is assigned to the instrument with the highest power at that bin. We mapped these spectrogram masks back into the linear-frequency spectrogram domain, filtered the complex spectrogram based on the masks, and computed the inverse filtered spectrogram using the original phase. The separation of the two instruments in the music is shown in Fig. 4. Informal listening test indicated, that the NMF2D algorithm was able to separate the two instruments very well.

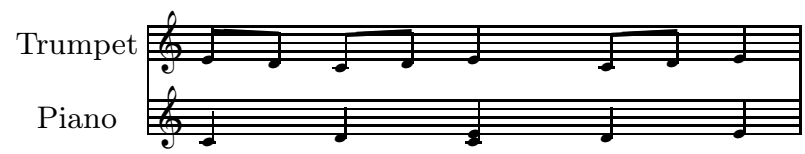

Fig. 1. Score of the piece of music used in the experiments. The music consists of a trumpet and a piano which play different short melodic passages each consisting of three distinct notes.

\section{Discussion}

In the previous section we compared the proposed NMF2D algorithm with NMFD. Both the NMF2D and the NMFD representation can be used to separate the instruments. However, since the notes of the individual instruments are spread over a number of factors in the NMFD, these must first be grouped manually or by other means. The NMF2D algorithm implicitly solves the problem of grouping notes.

If the assumption holds, that all notes for an instrument is an identical pitch shifted time-frequency signature, the NMF2D model will give better estimates of these signatures, because more examples (different notes) are used to compute each time-frequency signature. Even when this assumption does not hold, it migth still hold in a region of notes for an instrument. Furthermore, the NMF2D algorithm might be able to explain the spectral differences between two notes of different pitch by the 2-D convolution of the time-frequency signature.

Both the NMFD and NMF2D models perfectly explained the variation in the spectrogram. However, the number of free parameters in the two models are quite 


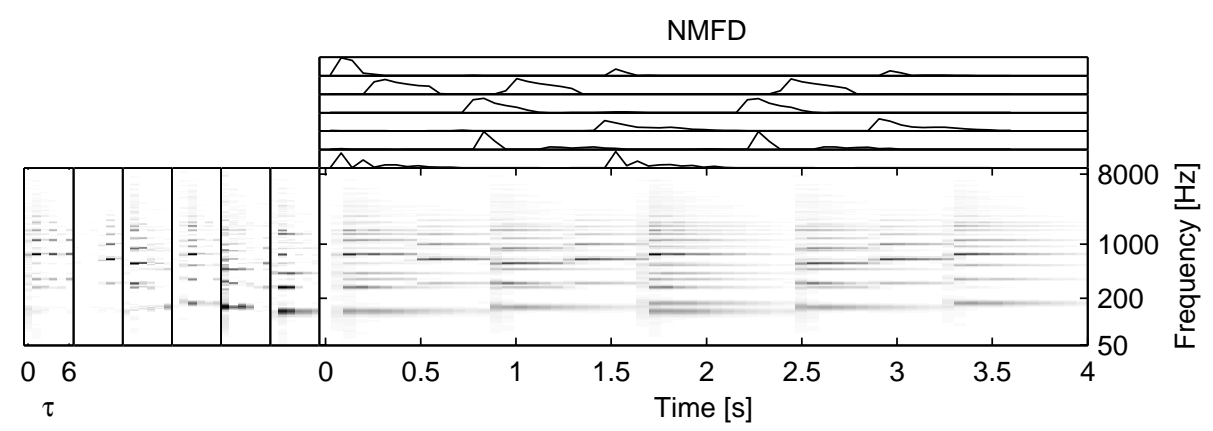

Fig. 2. Factorization of the piece of music using NMFD. The six time-frequency plots on the left are $\mathbf{W}^{\tau}$ for each factor, i.e. the time-frequency signature of the distincts tone played by the two instruments. The six plots on the top are the rows of $\mathbf{H}$ showing how the individual instrument notes are placed in time. The factors have been manually sorted so that the first three corresponds to the trumpet and the last three correspond to the piano.

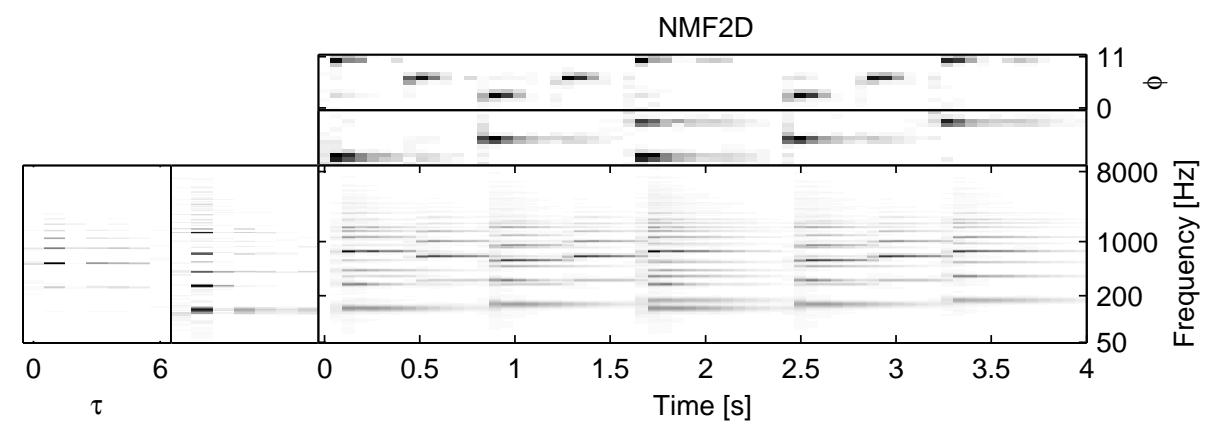

Fig. 3. Factorization of the piece of music using NMF2D. The two time-frequency plots on the left are $\mathbf{W}^{\tau}$ for each factor, i.e. the time-frequency signature of the two instruments. The two time-pitch plots on the top are $\mathbf{H}^{\phi}$ for each factor showing how the two instrument notes are placed in time and pitch.

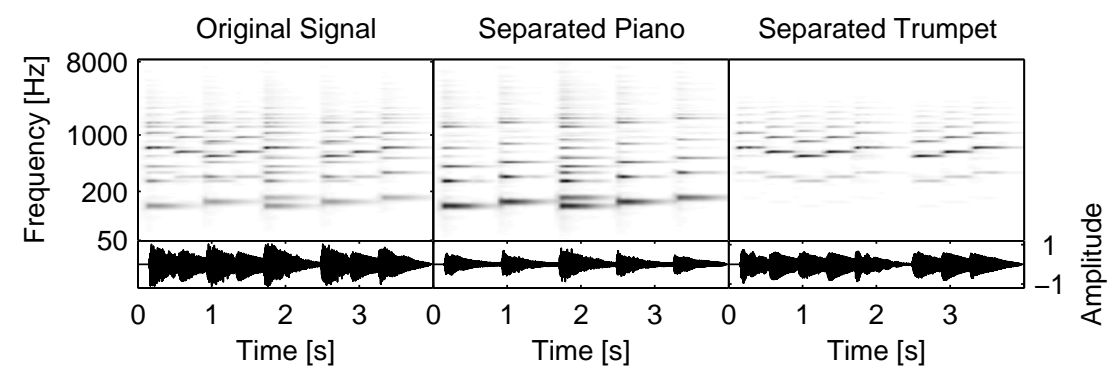

Fig. 4. Single channel source separation using NMF2D. The plots show the logfrequency spectrogram and the waveform of the music and the separated instruments. 
different. If the dimensionality of the spectrogram is $I \times J$, and $n_{\tau}, n_{\phi}$ denote the number of convolutive lags in time and pitch, NMFD has $\left(n_{\tau} I+J\right) \cdot d=$ $(7 \cdot 175+63) \cdot 6=7728$ parameters whereas NMF2D has $\left(n_{\tau} I+n_{\phi} J\right) \cdot d=$ $(7 \cdot 175+12 \cdot 63) \cdot 2=3962$ parameters. Consequently, the NMF2D was more restricted making the NMF2D the best model from an Occam's razor point of view.

Admittedly, the simple computer generated piece of music analyzed in this paper favors the NMF2D algorithm since each instrument key is a simple spectral shift of the same time-frequency signature. However, even when we analyze real music signals the NMF2D also gives very good results. Demonstrations of the algorithm for different music signals can be found at www.intelligentsound.org.

It is worth noting, that while we had problems making the NMFD algorithm converge in some situations when using the updates given by Smaragdis [7], the updates deviced in this paper to our knowledge always converge.

In the experiments above we used the NMF2D based on least squares. However, using the algorithm based on minimizing the KL divergence gave similar results. It is also worth mentioning that the NMF2D analysis is computationally inexpensive; the results in the previous section took approximatly 20 seconds to compute on a $2 \mathrm{GHz}$ Pentium 4 computer.

It is our belief that the NMF2D algorithm can be useful in a wide range of areas including computational auditory scene analysis, music information retrieval, audio coding, automatic music transcription, and image analysis.

\section{References}

1. Paatero, P., Tapper, U.: Positive matrix factorization: A non-negative factor model with optimal utilization of error estimates of data values. Environmetrics 5(2) (1994) $111-126$

2. Lee, D., Seung, H.: Learning the parts of objects by non-negative matrix factorization. Nature 401(6755) (1999) 788-91

3. Donoho, D., Stodden, V.: When does non-negative matrix factorization give a correct decomposition into parts? NIPS (2003)

4. Lee, D.D., Seung, H.S.: Algorithms for non-negative matrix factorization. In: NIPS. (2000) 556-562

5. Helén, M., Virtanen, T.: Separation of drums from polyphonic music using nonnegative matrix factorization and support vector machine. In: 13th European Signal Processing Conference. (2005)

6. Smaragdis, P., Brown, J.C.: Non-negative matrix factorization for polyphonic music transcription. IEEE Workshop on Applications of Signal Processing to Audio and Acoustics (WASPAA) (2003) 177-180

7. Smaragdis, P.: Non-negative matrix factor deconvolution; extraction of multiple sound sourses from monophonic inputs. International Symposium on Independent Component Analysis and Blind Source Separation (ICA) 3195 (2004) 494

8. Wang, B., Plumbley, M.D.: Musical audio stream separation by non-negative matrix factorization. In: Proceedings of the DMRN Summer Conference. (2005)

9. Virtanen, T.: Separation of sound sources by convolutive sparse cod. SAPA (2004) 